\title{
Type A and B Personalities From a Psychological Perspective Among Medical Students
}

\author{
Abdul Rahman Alfulaij \\ University of Bahrain, Bahrain
}

\author{
Faisal Abdullatif Alnasir \\ Arabian Gulf University, Bahrain
}

\begin{abstract}
Introduction: Research in the last two decades has shown that people with Type A personality are astronomically competitive, self-critical of performances, endeavor for perfectionism in their vocations, and strive toward goals in their lives without feeling a sense of bliss. Type A individuals will eventually suffer from cardiovascular diseases and heart attacks at an earlier age compared with those with Type B personality who are more inclined to be genial, relaxed, and internally fixated on their feelings and emotions. The main objective of this study was to ascertain the prevalence of Type A/B personalities among a first-year students who opt to study medicine. Methods: Seventy-seven year one medical students registered in the Arabian Gulf University, Bahrain during the academic year 2013-2014 were randomly selected to be involved in this study. Students completed a pre-designed questionnaire that included measures for Type A and Type B behaviors by using JAS (the Jenkins Activity Survey). Results: The number of female and male students was 49 (63.6\%) and 28 (36.4\%) respectively. Their ages ranged between 17 and 20 years with a mean of 17.8 years. Six different Arab nationalities were represented. The study found that the students with Type A personality exceeded those with B (59 (76.6\%) vs. 18 (23.4\%)). No significant relationship was found between personality type and other demographic characteristics except for a mild significant relationship $(p<0.053)$ with sex with more females having Type A than males. Conclusion: The majority of the studied medical students are of Type A personality betokening that such future careers, such as medicine, require extensive commitment and are opted conventionally by students who have Type A personalities. It was also found that more females have Type A than B personality characteristics, a finding that is contrary to the prevalent understanding of the Arab-Islamic society in the context that Arab women are oppressed and less decisive about their future.
\end{abstract}

Keywords: Type A/B personalities, masculine culture, medical students, JAS (the Jenkins Activity Survey), Arab-Islamic culture, traditions

\section{Introduction}

During the past two decades, Type A and Type B personality behavioral patterns have been the subject of excruciating investigations by medical and psychological researchers worldwide. Medical research has found that people who possess the hard-driving, competitive, time-urgent Type A pattern of behaviors are approximately twice as liable to develop coronary heart disease as are their more relaxed, easygoing Type B

Abdul Rahman Alfulaij, Ph.D., associate professor, University of Bahrain.

Faisal Abdullatif Alnasir, Ph.D., professor, Department of Family \& Community Medicine, College of Medicine and Medical Sciences, Arabian Gulf University. 
counterparts (Haynes, Feinleib, \& Kannel, 1990; Rosenman, Brand, Jenkins, Friedman, Status, \& Wurm, 2004; Dembroski, Weiss, Shields, Haynes, \& Feinbleip, 2007; Review Panel, 2002; Siegal, 2001). Albeit the precise physiological mechanisms that predispose them to Type As and Bs is obscure, their behavioral, psychological, and physiological responses to stress appear to differ. Accordingly, psychological research has endeavored to unearth the cognitive and motivational differences between Type A and B that lead them to interpret and respond to situations differently. Currently, there are several explanations concerning the underlying psychological dynamic that gives rise to Type A and B patterns and therefore, there is a desideratum to provide empirical evidence for the role of these personality types that can play a role in the health of the individual (Siegal, 2001).

Korotkov et al. (2011) carried out a study in 2010 to test the hypothesis verbalizing that Type B individuals would engage in more preventive acts than Type A. Two hundred medical students were administering the JAS (the Jenkins Activity Survey) for Type A/B personality. As predicted, in six out of seven regression analyses, Type B subjects were found to be engaged in more preventive, and fewer risk-related behaviors under high stress, than those who self-reported as Type A. Moreover, many studies have documented the relationship between the personality type and the presence of illnesses. To evaluate the presence of Type A in patients affected by OAG (Open-Angle Glaucoma) and the possible role of psychophysiological and psychosocial stresses as risk factors for OAG, R. M. Bubella, D. M. Bubella, and Cillino in 2012 studied 50 patients affected by OAG who underwent ophthalmologic examination consisting of complete bio-microscopy, tonometry and daily tonometric curve examination of the visual field by means of computerized perimeter "Octopus 1-2-3", and Type A/B personality JAS questionnaire. The results showed that $64 \%$ of the subjects had a high level of Type A behavior. It was concluded that personality evaluation is an astronomically consequential part for the diagnostic work-up and treatment process of OAG.

In another study, the prevalence of personality Types A and B in relation to individuals with MS (multiple sclerosis) and their demographic characteristics was investigated. It was found that $65 \%$ of the subjects were of Type A personality and that their behavioral pattern reported having more stress, nervousness and exigent tendencies. It was concluded that MS patients had more characteristics of Type A than type B (Shaygannejed et al., 2013).

For many students, admission to medical schools is considered the fulfillment of a long-held dream. The path to medical school always involves a great deal of apprehensiveness as it often requires competition and a drive to be the best in the school. Since the CMMS (College of Medicine and Medical Science) of the AGU (Arabian Gulf University) is a problem-based medical school with a community-oriented curriculum, students are expected to work in teams and learn in small groups (Alnasir Faisal \& Jaradat Ahmed, 2013). During the first academic year, students are required to take a course in medical psychology where they are taught the art of medicine cumulated with the principles of behavioral medication. They learn topics ranging from patient behavior and patient-physician interactions to the social and cultural issues in health care, not only learning the medical model of patient practice but rather the larger model of medical-psychosocial and moral development of practicing medicine. Such factors put great pressure on the students in trying to show their personal best, rather than compete with peers.

Students are taught that a physician's time is very precious. It is difficult to believe that the needs of any patient can be met adequately when physicians are encouraged and sometimes required to focus on the "clock". However rushed, the physician may feel for whatever reason that he or she must be aware that the 
sense of time urgency is anti-ethical to professional obligations. In general, patients know when they are being rushed along and can recognize when the physician's mind is not entirely with them. Some react with resentment and others by trying to extricate the physician from the situation. This may take the form of omitting critical symptoms from their statement of the problem, failing to ask questions that could clarify an instruction, or simply canceling a future appointment out of a genuine, albeit somewhat masochistic desire not to overburden the physician. Truly, none of these reactions is acceptable to the medical community and all can simply be avoided if the Type A personality of impatience and irritability that medical communities and environments often impose is controlled early enough for the sake of the health and well-being of the physicians.

One of the issues that students are exposed to is physician well-being. Medical students and physicians frequently give excellent advice to their patients about healthy lifestyle issues. However, a number of research studies have documented that medical profession personnel are likely to ignore such good advice when it comes to their own life (Carmelli \& Swan, 1996; Levine \& Sandine, 2005). Students at the beginning of the semester are subjected to Type A and Type B personality inventory test. By taking this test, students find out their style of dealing with daily issues, how they would communicate with their patients and whether they can have a more productive happier life cycle once they reach their mid-life crises (Carmelli \& Swan, 1996).

\section{Method}

Seventy-seven year one medical students (49 females, 28 males) out of 180 were recruited randomly to participate in this study of personality types. These students were registered in the CMMS of the AGU, Bahrain, during the academic year 2013-2014. None of the subjects had prior knowledge about the nature of the study and the details of the procedure. Students completed a questionnaire battery that included measures for Type A and Type B behaviors by using the short form of the JAS (with prior permission from the psychological corporation) which contained 11 multiple-choice item questionnaires. These questions assessed the students' everyday behaviors reflective of the Type A/B patterns. For example, it asked participants to report on the speed of pacing activities (e.g., "Has your spouse or your friend ever told you that you eat too fast?"; "How often do you bring work home with you as study materials related to your causes?"). Concern with deadlines (e.g., "If you tell someone you will meet them somewhere at a definite time, how often do you arrive late?"), and temper ("How was your temper when you were younger?"). At the conclusion, the results were scored while ensuring the subjects' confidentiality. Scores were assigned to the responses and those subjects who scored equal to or above the median (five) on the scale were classified as Type A whereas those who scored less the median were classified as Type B.

JAS is one of the most widely used methods of assessing Type A behavior. JAS was developed by David Jenkins, Stephen Zyzanski, and Ray Roseman in 1974 in an attempt to duplicate the clinical assessment of the Type A behavior pattern by employing an objective psychometric procedure (Yarnold, Mueser, Grau, \& Grimm, 1996). It was reported that individuals displaying a Type A behavior pattern are characterized by extremes of competitiveness, striving for achievement and personal recognition, aggressiveness, haste, impatience, explosiveness, and loudness in speech, characteristics that JAS attempts to measure. The 44 item long form student JAS (SJAS) used in locating students on the Type A/B personality continuum, requires an average of an additional ten minutes to complete than the short form $\mathrm{C}$ containing only the eleven scored items. 
Studies were conducted to determine whether the long and short forms provide comparable score distributions and factor structures for independent random samples of undergraduate students. Analysis revealed that the short form provides factors that are more independent than those provided by the long form of SJAS (Bryant \& Yarnold, 2012). The reliability of the two versions of JAS was affirmed by various studies. The first study done on 1,810 undergraduates revealed that estimates of the internal consistency (Chronbach's alpha) of the JAS and its subscales (hard-driving/competitive and speed impatience) ranged from moderately low to moderately high. In the second study, independent samples of undergraduate students completed the JAS at two testings, separated by two weeks $(n=137)$ or three months $(n=124)$, and revealed that the test-retest reliability of the JAS total score ranged from moderately to extremely high. The results of these studies suggested that the short version of JAS comprised of 11 items has satisfactory internal consistency and excellent test-retest reliability and support its continued use in research on these psychometric grounds. Concurrent validity of the JAS scores has also been established as well (Yarnold et al., 1996).

\section{Results}

Out of the 77 year-one medical students, 49 (63.6\%) were females and 28 (36.4\%) were males. Such a finding reflects the recent trend in demographic changes as the number of female students exceeds the number of male students. Student ages ranged between 17 and 20 years with a mean of 17.8 years. The females were older than the males ( $\geqslant 18$ years of age $80 \%$ vs. 57\%) (see Table 1). Students belonged to six different Arab nationalities, with the majority being Kuwaiti (36\%).

The score obtained in response to the JAS survey ranged between 1 and 11 with a mean of 5 (standard deviation $=2.616)$. Seventy-seven percent $(n=59)$ obtained a score of $\geqslant 5$ and were considered Type A personality while $23 \%(n=18)$ had less than five who were Type B. Responses to the different items in the JAS survey is highlighted in Table 2. The item analysis of the responses revealed that $74 \%(n=57)$ responded "Yes" to question 3 "I consider myself to be more stronger and competitive", 73\% $(n=56)$ responded "Yes" to question 2 "Everybody who knows me considers me to be fidgety and competitive", $71 \%(n=55)$ responded "Yes" to item No. 10 "Compared to the average student at my college, I consider myself to be more responsible when it comes to finishing my duties" and 70\% $(n=54)$ responded "Yes" to question No. 1 "My daily life is filled with problems that always need immediate attention and solution".

It was also found that more females than males (69\% vs. 31\%) are of Type A personality. This finding was moderately significant ( $p$-value $<0.053$ ) (see Table 3). With regard to age variable, it was shown that of those who are considered Type A, 32\% were 17 years of age and $68 \%$ were 18 years or older (see Table 4 ). Type A personality was found to be more common in the Kuwaiti nationals (23\%) followed by the Bahrainis (24\%) when compared with other nationalities. However, this finding was not statistically significant $(p<0.130)$.

Table1

Tabulated Statistics: Sex vs. Age

\begin{tabular}{llllll}
\hline Age in years & 17 & 18 & 19 & 20 & Total \\
\hline Sex & & & & & \\
Male & 12 & 14 & 1 & 1 & 28 \\
Female & 10 & 34 & 5 & 0 & 49 \\
Total & 22 & 48 & 6 & 1 & 77 \\
\hline
\end{tabular}


Table 2

Response to Questions From 1 to 11

\begin{tabular}{lllllllllllll}
\hline Question No. & 1 & 2 & 3 & 4 & 5 & 6 & 7 & 8 & 9 & 10 & 11 \\
\hline No & 23 & 21 & 20 & 36 & 29 & 33 & 40 & 37 & 42 & 22 & 27 & 50 \\
Yes & 54 & 56 & 57 & 41 & 48 & 44 & 37 & 40 & 35 & 55 & 50 \\
Total & 77 & 77 & 77 & 77 & 77 & 77 & 77 & 77 & 77 & 77 & 77 & \\
\hline
\end{tabular}

Table

Tabulated Statistics: Score vs. Sex

\begin{tabular}{lccc}
\hline Sex & Male & Female & Total \\
\hline Score & & & \\
5 or more & 18 & 41 & 59 \\
less than 5 & 10 & 8 & 18 \\
All & 28 & 49 & 77 \\
\hline
\end{tabular}

Notes. Pearson Chi-Square $=3.739, d f=1, p$-value $=0.053$; Likelihood Ratio Chi-Square $=3.630, d f=1, p$-value $=0.057$.

Table 4

Tabulated Statistics: Score vs. Age

\begin{tabular}{lcll}
\hline Age & 17 & $\geqslant 18$ & Total \\
\hline Score & & & \\
5 or more & $19(32 \%)$ & $40(68 \%)$ & 59 \\
Less than 5 & $3(17 \%)$ & $15(83 \%)$ & 18 \\
Total & 21 & 55 & 77 \\
\hline
\end{tabular}

\section{Discussion}

This study showed more females being prone to have Type A personalities. The results do provide some evidence for the premise that, when females deal with the future and everyday challenges, they exhibit more Type A characteristics compared to their male counterparts. However, this finding must be interpreted in the context of the Arab-Islamic culture when it comes to the role of the women in the larger society (Stowasser, 2006; Smyth, 1990; Garrity, Kotchen, McKean, Gurley, \& McFadden, 2000; Nieuwkerk, 2010). There is a changing trend in Arab society with regard to the role of women. Historically, incorrect interpretation of Islamic laws and social norms negatively impacted the way women were perceived when it came to defining their roles (Mobarak \& Soderfeldt, 2010). Now we see more women are taking jobs on par with men in such areas as medicine ( $75 \%$ of the first year medical students at AGU are females), and law and engineering. In today's changing society with regards to women's rights and equality, it is clear that women are increasingly applying themselves and trying to prove to others that their life role involves more than household chores, raising children and preparing food for their husbands. Furthermore, the societal trends show an extra effort on the part of women to identify themselves, not only as housewives, but as an integral part of society (Nieuwkerk, 2010; Romirowsky, 2007).

The prevalence of Type A personality characteristics among the female medical candidates compared to the males could indicate the increased likelihood of them experiencing heart problems and cardiovascular diseases later in their mid-life career ladder. These findings also impact women's societal roles within the Arab-Islamic cultural context. The study's results show that female medical students have the need to excel and 
prove their significance and independence in a patriarchal society.

An item analysis from a psychological perspective on responses to the JAS found that Type As and Bs do differ in their desires and motivation when it comes to judging their abilities in performing daily routines. Type A's have higher desires to achieve in life, but also their locus of control is more internal than external. From a social psychological perspective, feeling in control of events is an important aspect of their personality.

Some studies showed that the prevalence of Type A personality characteristics tend to increase as people age and hold more responsible positions in their career ladder. Professional women are on par with men as they achieve higher level positions such as doctors, lawyers, and entrepreneurs (Carmelli \& Swan, 1996; Smyth \& Yarandi, 1992). These studies also indicate a positive relationship between age and sex with regard to Type A behavior pattern, i.e., women tend to be more type A's as they get older in their career ladders (Smyth \& Yarandi, 2009). Although such findings were determined in the present study, the relationships were not significant.

Type A personalities were found to be more common in the Kuwaiti and Bahrainis students, but not statistically significant. Although there is no known research in this field regarding the relationship between nationality and Type A/B personality, based on this study it is clear that nationality may not have any correlation to personality types.

The plausibility exists that since first-year medical students are new to the environment and have not been subjected to the rigor of medical school, it may be difficult to see any specific changes in their behaviors (Levine \& Sandine, 2005). Since the results of this study indicate that more Type A's than Type B's exist among first year medical students, it is recommended that a similar study be performed on medical students nearing completion of their studies to find out whether the type of pressures during the course of medical studies and/or students' level of maturity could influence any changes in the findings (prevalence of Type A personality).

When doctors are self-aware of their psychological needs, they can provide better services to their patients (Pishkin, Braggio, \& Lovallo, 1987; Lovallo, Pincomb, Edward, Brackett, \& Wilson, 1986). While most doctors attend to the rigors of the medical profession, they often fail to schedule relaxation time for themselves and frequently internalize the burden of their patients whereby affecting relationships with their own family and friends (Mobarak \& Soderfeldt, 2010; Lovallo et al., 1986; Wright, Contrada, \& Glass, 2010).

\section{Conclusion}

The Type A/B personality inventory can be a useful tool for identifying aspects of medical students' characteristics in order for them to improve work efficiency, decrease anxiety, increase productivity, and improve doctor-patient communication in the future.

Since first-year medical students are more likely to have Type A behavioral patterns, it is recommended that medical students be educated regarding the implications and be able to take a break from their daily routines. They can improve their psychological fitness in the forms of social, religious, mental and/or family counseling whenever they feel the stress of their medical education (Lovallo et al., 1986; Hampton, 2006; Epstein \& Hundert, 2009).

A common perception in the medical profession is that the more relaxed Type B person often achieves 
more than the Type A because the Type B's are more open to creative ideas and new information than the hard-driving type A (Lovallo et al., 1986; Wright et al., 2010). Treatment of a Type A person would be the same as the treatment of any patient suffering from stress-related problems. It would typically involve teaching them some form of deep relaxation plus a cognitive re-evaluation of his/her life goals and of things that give him/her satisfaction (Pishkin et al., 1987; Lovallo et al., 1986; Hampton, 2006).

\section{References}

Alnasir Faisal, \& Jaradat, Ahmed. (2013). Prediction of medical students' performance in the medical school. Family Med. Medical Sci. Res., 2, 113. doi: 10.4172/2327-4972.1000113

Bryant, F. B., \& Yarnold, P. R. (2012). A measurement model for the short form of the student Jenkins Activity Survey. Journal of Personality Assessment, 1, 188-191.

Bubella, R. M., Bubella, D. M., \& Cillino, S. (2012). Type A behavior pattern: Is it a risk factor for open-angle chronic Glaucoma? Journal of Glaucoma, 230, 59-84.

Carmelli, D., \& Swan, G. E. (1996). The relationship of Type A behavior and its components in an elderly group of men from Western Group study. Psychosomatic Res., 40(5), 475-483.

Dembroski, T. M., Weiss, S. M., Shields, J. L., Haynes, S. G., \& Feinbleip, M. (Eds.). (2007). Coronary-prone behavior. New York; springer-Verlag.

Epstein, R. M., \& Hundert, E. M. (2009). Defining and assessing professional competence. Journal of the American Medical Association, 287, 226-235.

Garrity, T. F., Kotchen, J. M., McKean, H. E., Gurley, D., \& McFadden, M. (2000). The association between Type A behavior and changes in coronary risk factors among young adults. American Journal of Public Health, 80(11), 1354-1357.

Hampton, T. (2006). Experts address risk of physician suicide. Journal of the American Medical Association, 294, 1189-1191.

Haynes, S. G., Feinleib, M., \& Kannel, W. B. (1990). The relationship of psychological factors to coronary hearts disease in the Framingham study: III Eight year residence of coronary heart disease. American Journal of Epidemiology, III, 37-58.

Korotkov, D., Perunovic, M., Claybourn, M., Farser, I., Houlihan, M., Macdonald, M., \& Korotkov, K. A. (2011). The type B behavior pattern as a moderating variable of the relationship between stressor chronicity and health behavior. Journal of Health Psychology, 16(3), 397-409.

Levine, F. M., \& Sandine, E. (2005). Conceptualization in psychotherapy, the models approach. Lawrence Erlbaum Associates: Hillsdale, New Jersey.

Lovallo, W. R., Pincomb, G. A., Edward, G. L., Brackett, D. J., \& Wilson, M. F. (1986). Work pressure and Type A behavior pattern exam stress in male medical students. Psychosom Med., 48(1-2), 125-133.

Mobarak, A. F., \& Soderfeldt, B. (2010). Gender inequality in Saudi Arabia and its role in public service. East Mediterranean Health Journal, 16(1), 113-118.

Nieuwkerk, K. V. (2010). Women embracing islam: Gender and conversion in the West. University of Texas press.

Pishkin, V., Braggio, J. T., \& Lovallo, W. R. (1987). Physiological responses of Type A and Type B men during cognitive performance. J. Clinical Psychology, 43(3), 309-318.

Review Panel. (2002). On coronary-prone behavior and coronary heart disease: A critical review. Circulation.

Romirowsky, A. (2007). Fatwa rules to live by. Political Studies Review, 19(1/2), 174-176.

Rosenman, R. H., Brand, R. J., Jenkins, C. D., Friedman, M., Status, R., \& Wurm, M. (2004). Coronary heart disease in the western collaborative group study; final follow-up experience of 8.5 years. Journal of the American Medical Association, 233, 872-877.

Shaygannejed, V., Dehnavi, Sr., Ashtari, F., Karimi, S., Dehghani, L., Meamar, R., \& Tolou-Ghamari, Z. (2013). Study of Type A and $\mathrm{B}$ behavior patterns in patients in an Iranian population. International Medicine, 237, 76-82.

Siegal, J. M. (2001). Type a behavior. Epidemiologic Foundations and Public Health.

Smyth, K. A. (1990). Relationships among the variables age, weight, A/B behavior and sex. Journal of Human Hypertensions, 4(2), 116-118. 
Smyth, K. A., \& Yarandi, H. N. (1992). Type A/B behaviors in employed Black women. J. National Black Nurses Association, $6(1), 29-41$.

Smyth, K. A., \& Yarandi, H. N. (2009). A path model of Type A and Type B responses to coping stress in black women. Nurses Research, 41(5), 260-265.

Stowasser, B. F. (2006). Women in the Qur'an, traditions, and interpretation. Oxford University Press.

Wright, R. A., Contrada, R. J., \& Glass, D. C. (2010). Psychophysiology correlates of Type A behavior. In E. S. Katkin, \& S. B. Manuck (Eds.), Advances in behavioral medicine. Greenwich, C.T.: JAI Press.

Yarnold, P. R., Mueser, K. T., Grau, B. W., \& Grimm, L. G. (1996). The reliability of the student version of the Jenkins Activity Survey. Journal of Behavioral Medicine, 9(4), 401-414. 